\title{
La dinámica oceanográfica frente al Humedal Nacional Térraba-Sierpe y su relación con la muerte del manglar
}

Omar G. Lizano

Proyecto Hidroeléctrico El Diquís, Instituto Costarricense de Electricidad, Costa Rica; omar.lizano@ucr.ac.cr

\author{
11-VIII-2014. Corregido 20-XI-2014. Aceptado 22-XII-2014.
}

\begin{abstract}
The oceanographic dynamic in front of Térraba-Sierpe National Wetland (HNTS) and its relation with the mangrove's death. The Térraba-Sierpe Reserve has the largest mangrove in Costa Rica but has suffered from changes in sea level. I used published data to analyze the area and found that sea level rise, generated by coast subsidence or global warming, created a new platform for waves and changed the beach profile and sediment type. Increased sediment deposition on the north maintains the balance of this system, with a sediment gain on the island in front of the Térraba river mouth. A lower sediment output from the Sierpe river is generating a landward delta migration, especially in the southern part, a symptom of local sea level rise. Future scenarios of greater wave energy and additional sea level rise will not allow a dynamic sediment balance, especially on the southern part of the delta, and will generate a loss mangrove cover. Rev. Biol. Trop. 63 (Suppl. 1): 29-46. Epub 2015 April 01.
\end{abstract}

Key words: Coastal erosion, sea level rise, Térraba-Sierpe, wave refraction, mangrove.

La comunidad científica internacional ha declarado que la mayoría de los bordes costeros mundiales, están en estado de erosión (Dickson, Walkden \& Hall, 2007; Cooper, Beevers \& Oppenheimer, 2008; Mokrech et al, 2008). A nivel mundial se estima que un $70 \%$ de las playas de arena están retrocediendo (Gornitz, 1995). Islas y Cayos en el Océano Pacífico han sido declaradas en riesgo de inundación por el aumento del nivel del mar (Leatherman, 1977). Esto está relacionado con el calentamiento global producto de actividad antropogénica, en el que el derretimiento de los casquetes polares y la expansión térmica del agua, contribuyen cada vez más con mayores niveles del mar (Nicholls \& Cazenave, 2010). Pero también hay otras componentes mareográficas y atmosféricas que están cambiando. Se sabe que en algunas regiones está aumentando la velocidad de los vientos (Anónimo, 2010; Young, Zieger \& Bahanin, 2011), lo cual aumenta la altura de las olas (Lizano, 2007; Lizano, 2013).
También se pronostica una mayor intensidad de los ciclones tropicales (Knutson, Tuleya \& Kurihara, 1998; Pielke, Landsea, Mayfield, Laver \& Pasch, 2005; Alfaro, 2007). Esto implica una mayor altura de ola, que junto con una mayor marejada de huracán (apilamiento de agua sobre las costas, Sorensen (1978), hay mayor impacto tierra adentro (Lizano \& Lizano, 2010). El fenómeno de El Niño es otra componente mareográfica que aumenta el nivel del mar, que junto con mareas astronómicas muy altas, producen los niveles del mar adecuados para que el oleaje inicie o acelere los procesos de erosión en una playa (Lizano, 1997; Lizano \& Salas, 2001). Un mayor impacto costero se puede generar cuanto estos fenómenos se superponen (Lizano, 1997; Lizano \& Salas, 2001; Lizano \& Gutiérrez, 2011). La frecuencia con que esto ha sucedido, podría estar cambiando (Grant, 1981; Fetzek, 2009). La realidad es que cada vez son más frecuentes la presencia de eventos extremos (Anónimo, 
2012; Miller, 2012) y la superposición de estos fenómenos juntos: El Niño, mareas extraordinarias, tormentas, oleajes extraordinarios, entre otros, están provocando los patrones que se están observando de cambio en el nivel del mar.

La tectónica de placas genera una geodinámica costera que también juega un papel importante en los procesos de erosión, pues contribuye con hundimientos o levantamientos en la costa. Estos procesos han sido identificados en nuestras costas, tanto en el Pacífico (Protti, Güendel \& Malavassi, 2001; Cárdenes, 2003; Denyer, Cárdenes \& Kruse, 2004; Protti, 2007), como en el Caribe (Amador, Chacón \& Lizano, 1994; Cortés, Soto-Soto \& Jiménez, 1994; Denyer, Arias \& Personius 1994). El reciente terremoto de Guanacaste (05 de setiembre del 2012), produjo levantamientos en la costas del Pacífico Norte de hasta $75 \mathrm{~cm}$ (Delgado \& Arguedas, 2012), pero hundimientos en el interior del Golfo de Nicoya (Marino Protti, com. pers.). Específicamente sobre el Golfo Dulce, el artículo de Hebbeln, Beese y Cortés (1996) indica que esta zona es tectónicamente muy activa. En esta confluyen la Placa Cocos y la Placa Nazca, las cuales se subducen en la Placa Caribe, lo cual ha estado levantado la costa a través de los últimos 80 millones de años. Pero además agrega que aunque el proceso de levantamiento aún se mantiene, la parte interna del golfo, sobre el borde norte, se está hundiendo debido a ajustes tectónicos locales. Espinoza et al. (2005) también agregan que en la génesis de los sismos se conocen dos fuentes principales, una de ellas ligada a la subducción de la Fosa Mesoamericana y a lo largo de la Fractura de Panamá, la otra ligada a fallas locales. Argumentan también, que esta sismicidad es la responsable de las deformaciones (neotectónicas) observadas en el delta del Térraba-Sierpe.

La región de HNTS contiene el bosque de manglar más grande de Costa Rica (Jiménez, 1994; Zamora, 2006; Reyes, Miranda, Monge \& Salas, 2007). Específicamente sobre el manglar, cambios geomorfológicos fueron señalados por Ortíz (2008). Este estudio asocia la pérdida de cobertura del manglar y la desaparición de islas en esta región, a los cambios del nivel del mar. Procesos similares apunta Cárdenes (2003) sobre la región de Parrita, y en Isla Damas. Espinoza et al. (2005) señalan que la pérdida del manglar es debido al reemplazo del sustrato de lodo por lodos arenosos, lo cual es indicativo de un proceso transgresivo en la zona. También señalan que el gran aporte de sedimentos del Río Grande de Térraba al norte, mantiene este sistema estuarino en un régimen de equilibrio entre los procesos marinos y fluviales, mientras que al sur, el menor aporte de sedimentos del Río Sierpe, podría estar influyendo en que el delta sea cada vez más afectado por la acción marina.

El objetivo de este trabajo es estudiar la dinámica del oleaje y de las mareas frente a la región de Sierpe-Térraba y la relación que esto tiene con los procesos de erosión y con la muerte del manglar, especialmente sobre la región sur de este sistema estuarino. Se hace un análisis descriptivo oceanográfico y físico del sector costero influenciado por las desembocaduras de los ríos Grande de Térraba y Sierpe sobre el HNTS.

\section{MATERIALES Y MÉTODOS}

Estadísticas de oleaje: Las características básicas del oleaje (altura, período y dirección) y el viento (magnitud y dirección) mar adentro (aproximadamente $30 \mathrm{~km}$ de la costa) correspondientes al Pacífico Sur de Costa Rica, se obtuvieron de los re-análisis de pronóstico de oleaje y viento de la NOAA. Los datos se suministran cada tres horas desde enero de 1997 hasta hoy día.

Las series de tiempo de los datos de la NOAA sobre características de oleaje (19972008), fueron filtradas según la dirección de la ola para identificar las frecuencias (número de veces) de ese oleaje en las direcciones N, NE, E, SE, S, NW, W $( \pm 22.5$ grados alrededor de la dirección) e identificar desde donde principalmente llega el oleaje (mar adentro), frente a la región de HNTS.

Para identificar distintos grupos de olas, se hizo una análisis de dispersión de la altura 
y período de ola, lo cual tiene relación con la generación o procedencia de estos oleajes (Lizano, 2007). Se separaron estos grupos para determinar sus principales características y propagar las olas hacia la costa con el modelo de oleaje (Lizano et al., 2001) hacia la costa.

Análisis de mareas: El análisis de las mareas se realizó utilizando el software Tides \& Currents (Lizano, 2006), que tiene predicciones mareales desde 1901 hasta el 2099. Este software tiene la capacidad de extraer datos minuto a minuto del nivel de la marea, los pleamares y bajamares, eventos extremos, a través del período deseado.

Dado que los distintos niveles de marea son importantes estudiarlos en esta región, se simularon las condiciones de oleaje en marea baja (nivel de sicigias, o cero del mar), para mostrar los efectos de los bancos de arena frente a las bocanas del manglar de esta región. También se simuló el oleaje con condición de mareas altas, que como se verá, son las que proporcionan la plataforma adecuada para que los oleajes ingresen hacia el interior del manglar.

Batimetría: La batimetría que abarcó la costa hasta aguas con profundidades mayores de $100 \mathrm{~m}$, se digitalizó desde la batimetría de las cartas náuticas del software MapSource de Garmín, y fue interpolada a una resolución de aproximadamente $400 \mathrm{~m}$. La batimetría de aguas poco profundas fue levantada con un ecosonda Garmin de doble frecuencia integrado a un GPS. Luego fue interpolada a un tamaño de rejilla de unos $124 \mathrm{~m}$ para usarla en el modelo de olas. Los contornos de la costa fueron digitalizados desde la imagen de Google Earth del 2005 sobre la cual se sobrepusieron los datos de batimetría. Los valores de batimetría fueron corregidos y ajustados al nivel de referencia de mareas de sicigias (nivel "0") (Lizano, 2006) para ese día con las predicciones de la marea en Puerto Quepos.

Modelación de oleaje: Se utilizaron los datos de la NOAA, series del re-análisis del modelo WAVEWATCH III que tienen características de las olas y el viento cada $3 \mathrm{~h}$ desde 1997. La línea de tendencia para la serie temporal de olas se determinó son el software SYSTAT, mediante el cual también se determinó la significancia de los estadísticos de "t" de Student y la razón " $F$ " de Ficher. El valor de estos estadisticos, $\mathrm{t}>2.0$ y $\mathrm{F}>4$, indicarían una tendencia positiva real o un estadístico robusto (Blank, 1980). La propagación de oleaje hacia el área de interés (HNTS) se realizó con un modelo numérico de oleaje de los llamados de tercera generación (SWAN: Simulating Waves Nearshores), Booij, Ris \& Holthuijsen, 1999; Lizano et al., 2001) en dos dominios: uno de agua profunda con resolución espacial de $413 \mathrm{~m}$ aproximadamente, y uno de aguas poco profundas, de resolución espacial de $124 \mathrm{~m}$ aproximadamente. En esta ocasión se usó una versión más actualizada (SWAN ver. 4072) que la de Lizano et al. (2001), que incluye refracción, difracción y reflexión de oleaje.

Geomorfología: Fotografías aéreas tomadas desde un helicóptero, se usaron para identificar regiones donde los árboles del manglar se están muriendo, o donde hay procesos de erosión. También se utilizaron fotos tomadas desde una embarcación de algunas regiones costeras que muestran erosión.

La Sección de Gestión Ambiental del ICE digitalizó los bordes de la costa e islas desde las fotografías aéreas disponibles desde 1948 hasta 1997. Se digitalizó adicionalmente la costa de la imagen del 2005 del Google Earth para tener la información más actual. Con estas imágenes y el software ArcGis 10, se determinó el área de las principales islas al frente de la región del HNTS. Con ello se realizó un balance de la variación de las áreas de las islas para identificar su variación, lo cual está relacionado a la dinámica de los sedimentos en la zona.

\section{RESULTADOS}

Área de estudio: El Humedal Nacional Térraba-Sierpe se localiza en el Pacífico Sur de Costa Rica (Fig. 1). Pertenece a la Provincia de Puntarenas. Está dentro de la Región Brunca, 


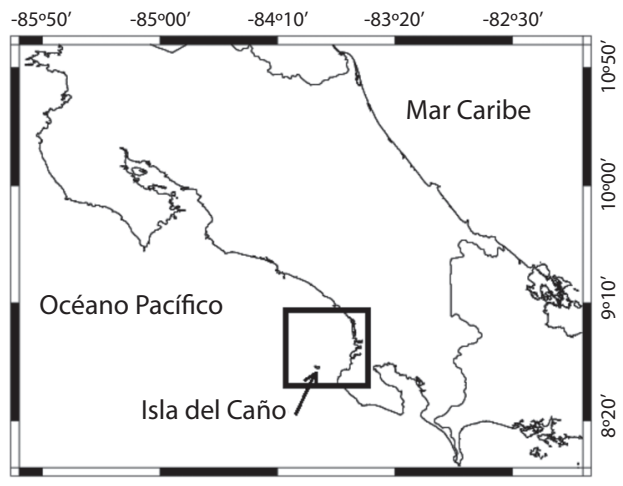

Fig. 1. Área de estudio. Contornos batimétricos en metros. Fig. 1. Study area. Bathymetric contours in meters.

y en la sub-región llamada El Diquís (Anónimo, 2006). Constituye un sistema deltaico formado por la deposición de sedimentos de los ríos Térraba y Sierpe (Ortiz, 2008), con un ecosistema forestal, principalmente manglar, que es inundado periódicamente por las mareas (Espinoza et al., 2005). La Fig. 1 corresponde aproximadamente a la región batimétrica utilizada en este estudio.

Estadísticas de oleaje: El análisis de frecuencia de la dirección de oleaje frente a la región del HNTS (Fig. 1), indica que el $62 \%$ es del suroeste (SW), un 33\% del sur (S) y un 3\% del oeste (W). El resto de las direcciones no aportan frecuencias importantes, por lo tanto no se tomarán en cuenta para el análisis de modelación de oleaje. Además, excepto oleaje del noroeste, las demás direcciones no son viables de modelación según la posición del área de estudio, pues no es razonable tener oleaje frente del HNTS de direcciones, por ejemplo del sureste.

Una vez identificada la frecuencia del oleaje en las direcciones de interés, se realizó un análisis de dispersión de altura y período para identificar los tipos de oleajes (mar de viento y marejada de fondo, Lizano (2007) que se pueden esperar al frente del HNTS. La Fig. 2 muestra el resultado gráfico de este análisis.
Se aprecia en las Figs. 1 y 2 que para cada dirección escogida, hay dos trenes de oleaje fácilmente distinguibles: uno de períodos de olas cortos y otro de períodos de ola más largos. Estos patrones de oleaje tienen relación con el origen: los oleajes de períodos cortos son de formación local, y los de períodos largos, de formación remota (Lizano, 2007).

Como hay distintos trenes de olas, se separaron esas series según sus bandas de períodos de ola, de manera que se pudiera determinar las características promedio y los valores mínimos y máximos de cada grupo. Estas características son importantes para realizar el estudio de modelación de oleaje. Estos valores se resumen en el Cuadro 1.

Del Cuadro 1 se resume que la altura promedio de las olas (mar adentro) en esta región están entre 1 y $1.7 \mathrm{~m}$, con períodos promedio entre 6 y $15 \mathrm{~s}$, y aunque la mayor frecuencia del oleaje es del suroeste, los oleajes del sur con períodos cortos (generados por tormentas locales), son las de mayor altura $(2.92 \mathrm{~m})$. El viento sin embargo, es en promedio mayor desde el oeste $(5.7 \mathrm{~m} / \mathrm{s})$, pero con máximos desde el suroeste $(13.7 \mathrm{~m} / \mathrm{s})$.

Análisis de mareas: Las principales características de la marea de Quepos (Lizano, 2006), la estación más cerca a la zona de estudio, se resumen en el Cuadro 2.

Si bien es cierto que las características de las mareas descritas anteriormente son útiles para establecer la dinámica del nivel del mar en el lugar, más importante para efectos de nuestro estudio de modelación de oleaje, serán los niveles máximos que se estén dando en el HNTS, pues el oleaje combinado con estos niveles, están asociados a los problemas de erosión en esta región, como discutiremos ampliamente más adelante. Se sabe que niveles superiores a $3.00 \mathrm{~m}$ en el Puerto de Puntarenas son los niveles sobre los cuales comienzan a inundarse la mayoría de regiones costeras de Costa Rica (Lizano \& Lizano, 2010). Como los ámbitos de la marea son distintos a lo largo de la costa (Lizano, 2006), se hace necesario identificar el nivel máximo de la marea en Quepos que 


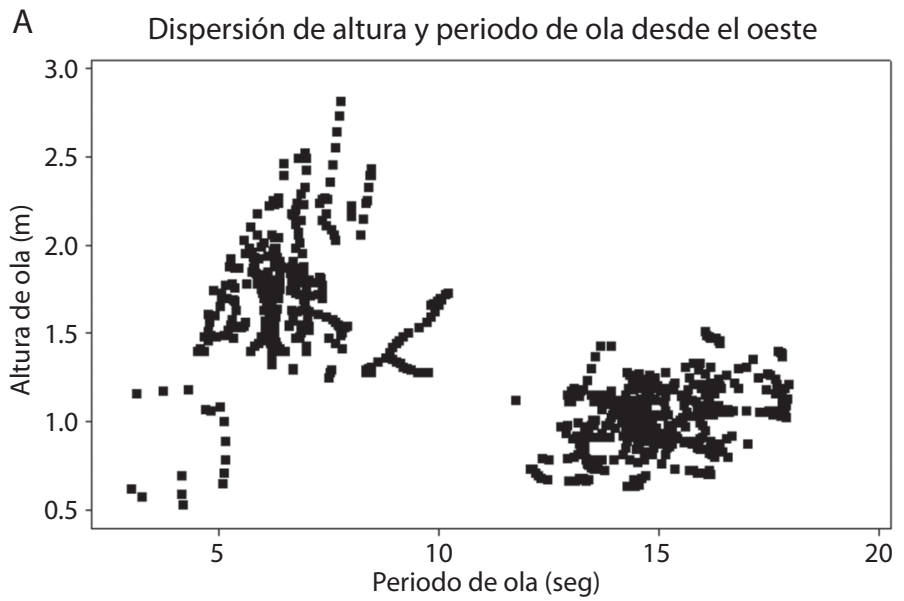

B Dispersión de altura y periodo de ola del suroeste

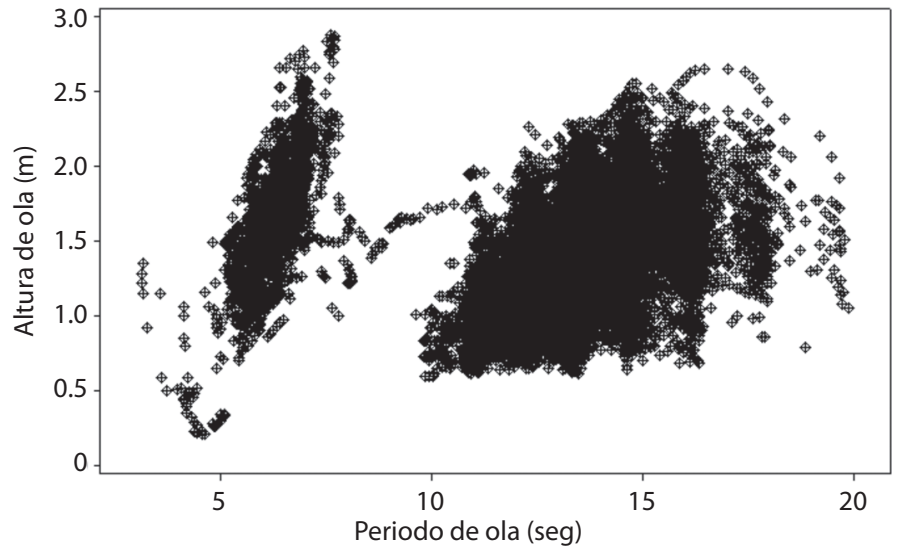

C Dispersión de altura y periodo de ola para la dirección sur

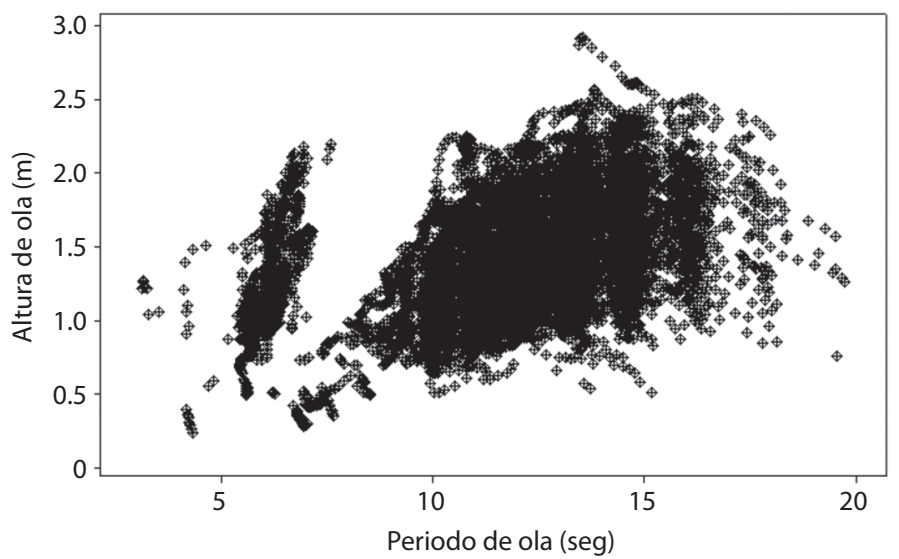

Fig. 2. Análisis de distribución de la altura (m) y período (s) de la ola al frente del HNTS en A: dirección oeste, B: dirección suroeste y C: dirección sur.

Fig. 2. Analysis of distribution of the wave height (m) and period (s) in front of HNTS in A: west direction, B southwesterly direction and $\mathrm{C}$ : south direction. 


\section{CUADRO 1}

Estadísticas del oleaje y viento para el análisis de modelación de oleaje

TABLE 1

Wave and wind statistics for the analysis of wave modeling

Pacífico Sur de Costa Rica, dirección oeste:

\begin{tabular}{lcccc} 
& \multicolumn{2}{c}{ Períodos menores de $7 \mathrm{~s}$} & \multicolumn{2}{c}{ Períodos mayores de $7 \mathrm{~s}$} \\
& Prom. & Máximo & Prom. & Máximo \\
$\mathrm{Hs}(\mathrm{m})$ & 1.72 & 2.81 & 1.02 & 1.73 \\
$\mathrm{AZ}\left({ }^{\circ}\right)$ & 254.79 & 292.09 & 274.95 & 292.45 \\
Tp $(\mathrm{s})$ & 6.29 & 7.93 & 14.89 & 17.98 \\
WIN $(\mathrm{m} / \mathrm{s})$ & 5.70 & 10.80 & 3.15 & 7.83 \\
DIRW $\left({ }^{\circ}\right)$ & 88.80 & 341.28 & 179.73 & 359.41 \\
\hline
\end{tabular}

Pacífico Sur de Costa Rica, dirección suroeste:

\begin{tabular}{lcccc} 
& \multicolumn{2}{c}{ Períodos menores de $8 \mathrm{~s}$} & \multicolumn{2}{c}{ Períodos mayores de $10 \mathrm{~s}$} \\
& Prom. & Máximo & Prom. & Máximo \\
Hs $(\mathrm{m})$ & 1.61 & 3.88 & 1.34 & 2.65 \\
$\mathrm{AZ}\left({ }^{\circ}\right)$ & 22.59 & 247.49 & 212.25 & 247.48 \\
Tp $(\mathrm{s})$ & 6.22 & 7.93 & 13.52 & 19.89 \\
WIN $(\mathrm{m} / \mathrm{s})$ & 4.45 & 13.70 & 3.58 & 11.04 \\
DIRW $\left({ }^{\circ}\right)$ & 90.86 & 359.89 & 136.87 & 360.00 \\
\hline
\end{tabular}

Pacífico Sur de Costa Rica, dirección sur:

\begin{tabular}{lcccc} 
& \multicolumn{2}{c}{ Períodos menores de $7 \mathrm{~s}$} & \multicolumn{2}{c}{ Períodos mayores de $7 \mathrm{~s}$} \\
Variable & Prom. & Máximo & Prom. & Máximo \\
$\mathrm{Hs}(\mathrm{m})$ & 1.19 & 2.20 & 1.46 & 2.92 \\
$\mathrm{AZ}\left({ }^{\circ}\right)$ & 192.36 & 202.50 & 196.07 & 202.50 \\
Tp $(\mathrm{s})$ & 6.21 & 7.99 & 12.77 & 19.74 \\
WIN $(\mathrm{m} / \mathrm{s})$ & 3.53 & 9.30 & 3.60 & 11.78 \\
DIRW $\left({ }^{\circ}\right)$ & 123.14 & 360.00 & 134.23 & 360.00 \\
\hline
\end{tabular}

Prom: promedio, Hs: altura de ola significativa, AZ: dirección de la ola (respecto al norte desde donde viene), Tp: período de la ola, WIN: velocidad del viento (a 10m de altura) y DIRW: dirección del viento (respecto al norte hacia dónde va).

\section{CUADRO 2}

Características de la altura (m) de marea en la estación de Puerto Quepos

TABLE 2

Features of the tide height $(\mathrm{m})$ at Puerto Quepos station

\begin{tabular}{cccccccccc}
\multicolumn{1}{c}{ Estación } & $\operatorname{Lat}\left({ }^{\circ}\right)$ & $\operatorname{Long}\left({ }^{\circ}\right)$ & RP & PA & MP & PP & PB & MM \\
Quepos & $09^{\circ} 24^{\prime}$ & $84^{\circ} 10^{\prime}$ & 2.07 & 2.56 & 1.28 & 2.31 & 0.24 & 3.07 \\
\hline
\end{tabular}

RP: rango promedio, PA: promedio de altamares, MP: marea promedio, PP: promedio de pleamares, PB: promedio de bajamares, MM: marea máxima (Lizano, 2006). 
corresponde a la marea en Puntarenas. Para esto se hizo uso del software Tides \& Currents (Lizano, 2006). El valor que corresponde al nivel de la marea de $3.00 \mathrm{~m}$ de Puntarenas, es $2.80 \mathrm{~m}$ para Quepos.

La Fig. 3 resume gráficamente el análisis de bajamares y pleamares en Puerto Quepos. Se distingue en la Fig. 3 que muchos valores de marea superan el nivel de inundación de $2.80 \mathrm{~m}$ para Quepos. Exactamente 975 veces se supera este valor en 20 años. Por otro lado, las mareas tienen ciclos máximos de 4-5 años (Lizano, 2006), en los que las mareas son extraordinarias (máximas de $3.07 \mathrm{~m}$, Fig. 4). Durante el ciclo de mareas astronómicas extraordinarias, con más frecuencia se supera el valor de $2.80 \mathrm{~m}$ (ejemplo: 1993, 1997, 1998, 2002, 2006, 2010, 2015 y 2020, Fig. 4), que cuando estamos en un ciclo de mareas astronómicas bajas (ejemplo: 2004 y 2008, Fig. 4).

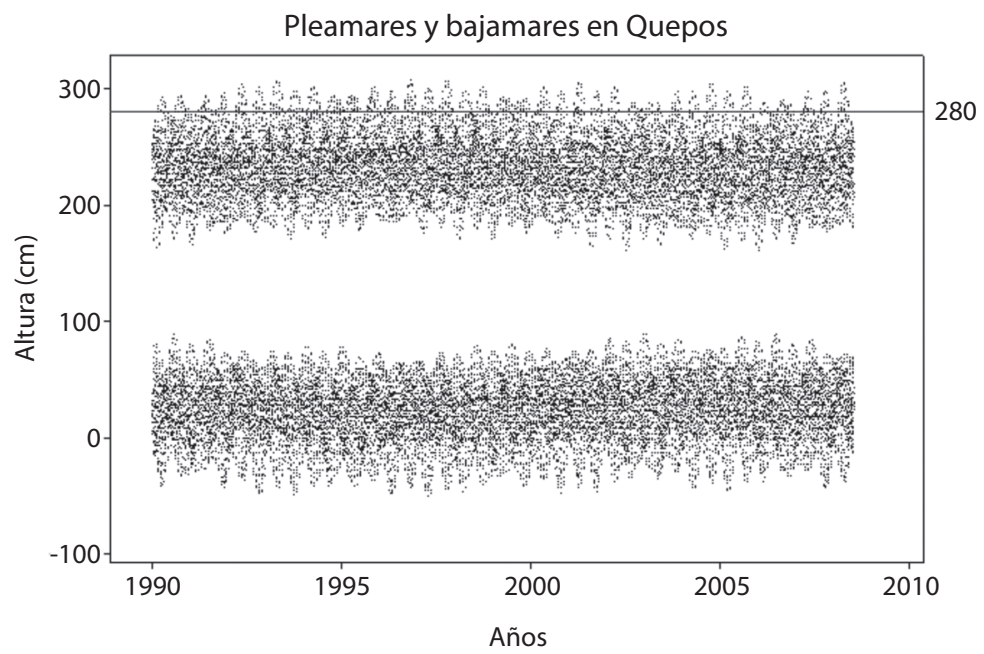

Fig. 3. Ámbitos de variación de las pleamares y bajamares en Quepos en 20 años.

Fig. 3. Variation range for high and low tides in Quepos in 20 years.

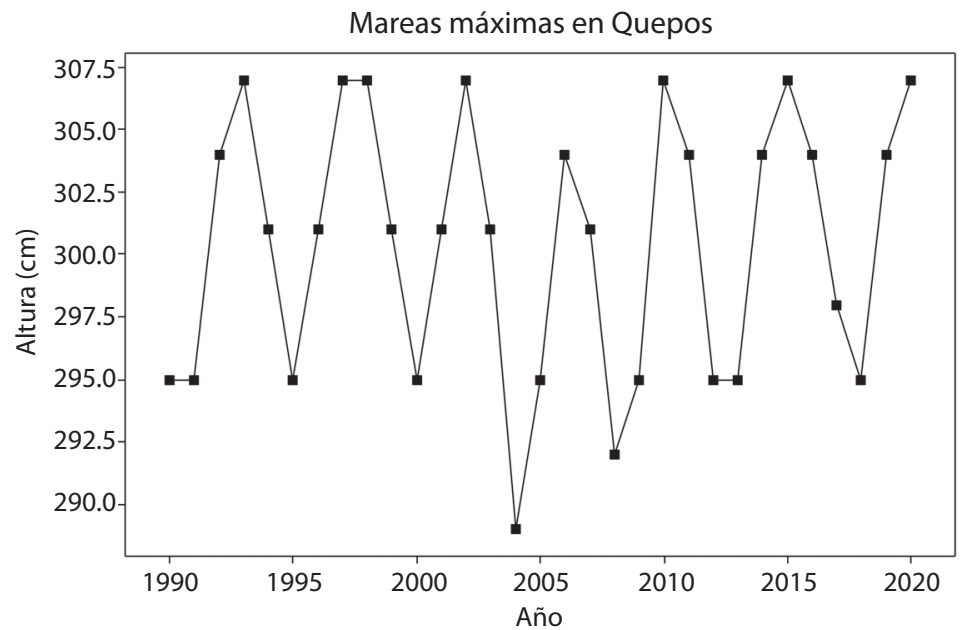

Fig. 4. Valores máximos de las mareas por año en Quepos. / Fig. 4. Maximun values of the tides per year in Quepos. 
Como el valor promedio de la serie de los 975 valores que superan 10 s $2.80 \mathrm{~m}$ en 20 años es $2.87 \mathrm{~m}$, se usará este valor como el nivel de marea astronómica alta probable, y que con bastante frecuencia, se presenta en la región el HNTS. Exactamente en 20 años se supera 397 veces el valor de $2.87 \mathrm{~m}$. Durante el año de 1998, 31 veces se superó este valor, y prácticamente todos los meses del año. En 2004 tan solo lo superó tres veces. En el 2010 se superó 24 veces, siendo esto prácticamente todos los meses en la segunda mitad del año.

Batimetrías para la propagación de oleaje hacia la costa: La propagación de oleaje hacia la costa se realizó a través la plataforma continental al frente de la región del HNTS en dos regiones. En la Fig. 5A se muestra la región de agua profunda, y el recuadro en esta corresponde al área de la región de agua poco profunda (Fig. 5B).

Modelación de oleaje desde agua profunda: Se realizó el estudio de modelación de oleaje sobre la región de agua profunda (Fig. 5A) con 30 condiciones de oleaje y viento promedio y valores máximos. Algunos casos específicos y representativos se discutirán a continuación.

Las condiciones promedio de la altura del oleaje de las dos direcciones más importantes en esta región, y que nos permiten identificar los patrones de los efectos principales de la batimetría sobre el oleaje, se muestran en la Fig. 6A y 6B.

Es de resaltar que el efecto de refracción de oleaje (cambio de dirección), va aumentado conforme el oleaje cambia de oeste, suroeste y sur respectivamente. Es decir, el oleaje entra más directamente a esta región desde el oeste; se reproduce muy bien el efecto de sombra (pérdida de altura de ola) detrás de Isla del Caño. Es evidente el efecto que ejerce la Isla del Caño, y la plataforma menos profunda al sur de ella, reduciendo la altura de oleaje que puede alcanzar la región del HNTS, especialmente desde la dirección sur.

La Fig. 6C y 6D muestran los resultados de la refracción del oleaje para la dirección principal de mayor frecuencia del oleaje: el suroeste. En este caso, el oleaje de períodos medios del suroeste $(\mathrm{Tp}=13.5 \mathrm{~s}$, Fig. $6 \mathrm{C})$ experimenta más
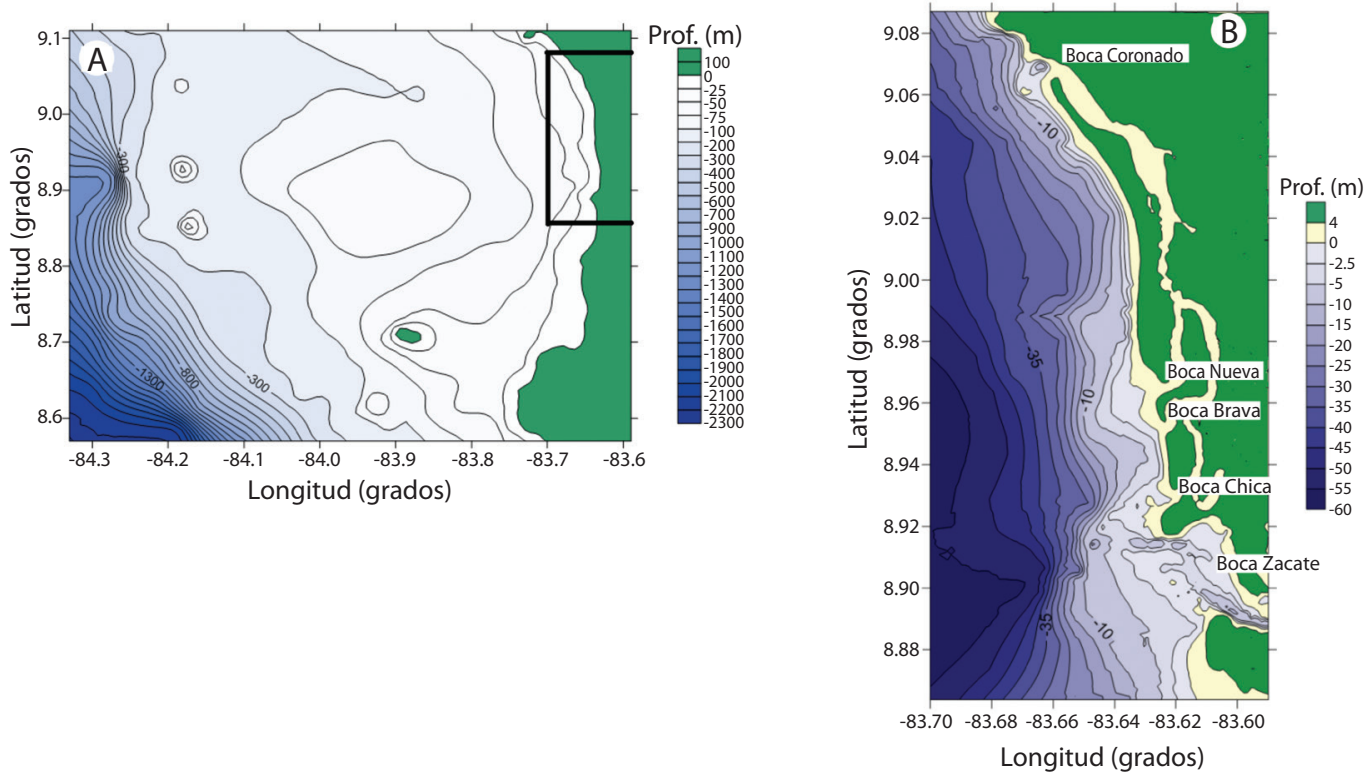

Fig. 5. A: batimetría $(\mathrm{m})$ de agua profunda y B: batimetría $(\mathrm{m})$ en agua poco profundas.

Fig. 5. A: bathymetry $(\mathrm{m})$ deep water and B: bathymetry $(\mathrm{m})$ in shallow water. 

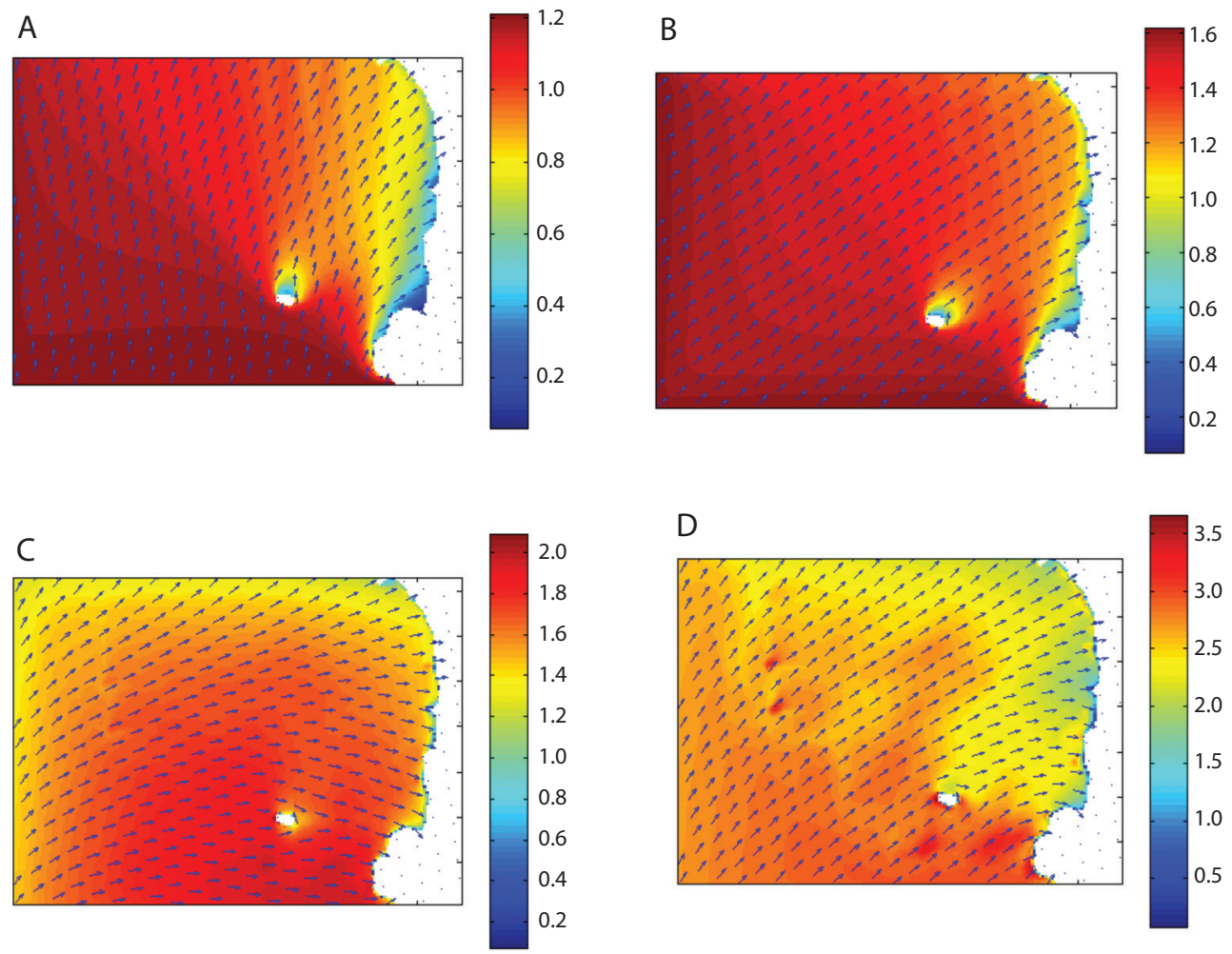

Fig. 6. Distribución de la altura $(\mathrm{m})$ y dirección (flecha) del oleaje desde el: $6 \mathrm{~A}$ : sur con $\mathrm{Hs}=1.2 \mathrm{~m}, \mathrm{Tp}=6.2 \mathrm{~s}, \mathrm{Viento}=11.0 \mathrm{~m} / \mathrm{s}$ y Direc. viento $=134^{\circ}, 6 \mathrm{~B}$ : suroeste con $\mathrm{Hs}=1.6 \mathrm{~m}, \mathrm{Tp}=6.5 \mathrm{~s}$, Viento $=4.5 \mathrm{~m} / \mathrm{s}$ y Direc. viento $=90^{\circ}, 6 \mathrm{C}$ : suroeste con $\mathrm{Hs}=1.3 \mathrm{~m}$, $\mathrm{Tp}=13.5 \mathrm{~s}$, Viento $=11.0 \mathrm{~m} / \mathrm{s}$ y Direc. viento $=140^{\circ}, 6 \mathrm{D}$ : suroeste con $\mathrm{Hs}=2.65 \mathrm{~m}, \mathrm{Tp}=20.0 \mathrm{~s}$, Viento $=11.0 \mathrm{~m} / \mathrm{s}$ y Direc. viento $=140^{\circ}$.

Fig. 6. Distribution of the wave height $(\mathrm{m})$ and direction (arrow) from: $6 \mathrm{~A}$ : south with $\mathrm{Hs}=1.2 \mathrm{~m}, \mathrm{Tp}=6.2 \mathrm{~s}$, Wind $=11.0 \mathrm{~m} / \mathrm{s}$ and Wind dir. $=134^{\circ}, 6 \mathrm{~B}$ southwest with $\mathrm{Hs}=1.6 \mathrm{~m}, \mathrm{Tp}=6.5 \mathrm{~s}$, Wind $=4.5 \mathrm{~m} / \mathrm{s}$ and Wind dir. $=90^{\circ}, 6 \mathrm{C}$ : southwest with $\mathrm{Hs}=1.3 \mathrm{~m}, \mathrm{Tp}$ $=13.5 \mathrm{~s}$, Wind $=11.0 \mathrm{~m} / \mathrm{s}$ and Wind dir. $=140^{\circ}, 6 \mathrm{D}$ : southwest with $\mathrm{Hs}=2.65 \mathrm{~m}, \mathrm{Tp}=20.0 \mathrm{~s}$, Wind $=11.0 \mathrm{~m} / \mathrm{s}$ and Wind $\operatorname{dir} .=140^{\circ}$.

refracción (cambio de dirección) sobre esta plataforma continental. También se observa que en la condición más severa de oleaje del suroeste (Fig. 6D ), las alturas de las olas pierden mucha energía (altura), por efecto de la Isla del Caño, y en cierto grado, por la saliente de la Península de Osa (extremo inferior derecho de la Fig. 6).

\section{Modelación de oleaje en agua poco pro-}

funda: El análisis de modelación en aguas poco profundas (Fig. 5B) se realizó propagando las condiciones del oleaje desde la región anterior (desde agua profunda, Fig. 5A). Se analizaron también, los 30 casos de combinaciones de altura, período, dirección de ola y viento que se simularon desde agua profunda. Se discutirán en este apartado los casos más representativos.

Dirección oeste: la Fig. 7A a la 7D muestran las características de la altura del oleaje y la dirección según la refracción del oleaje en agua poco profunda desde esta dirección.

Un análisis de los resultados anteriores, indica que el oleaje con alturas promedio $(\mathrm{Hs}=1.7 \mathrm{~m})$, de períodos bajos $(\mathrm{Tp}=6.4 \mathrm{~s})$ y en marea baja (Fig. 7A) pierde energía (altura) desde mar adentro (Cuadro 1), y rompe con altura del orden de $1 \mathrm{~m}$ frente a los bajos batimétricos de las bocas. Por otro lado, el oleaje con alturas promedio $(\mathrm{Hs}=1.7 \mathrm{~m})$ y marea alta 

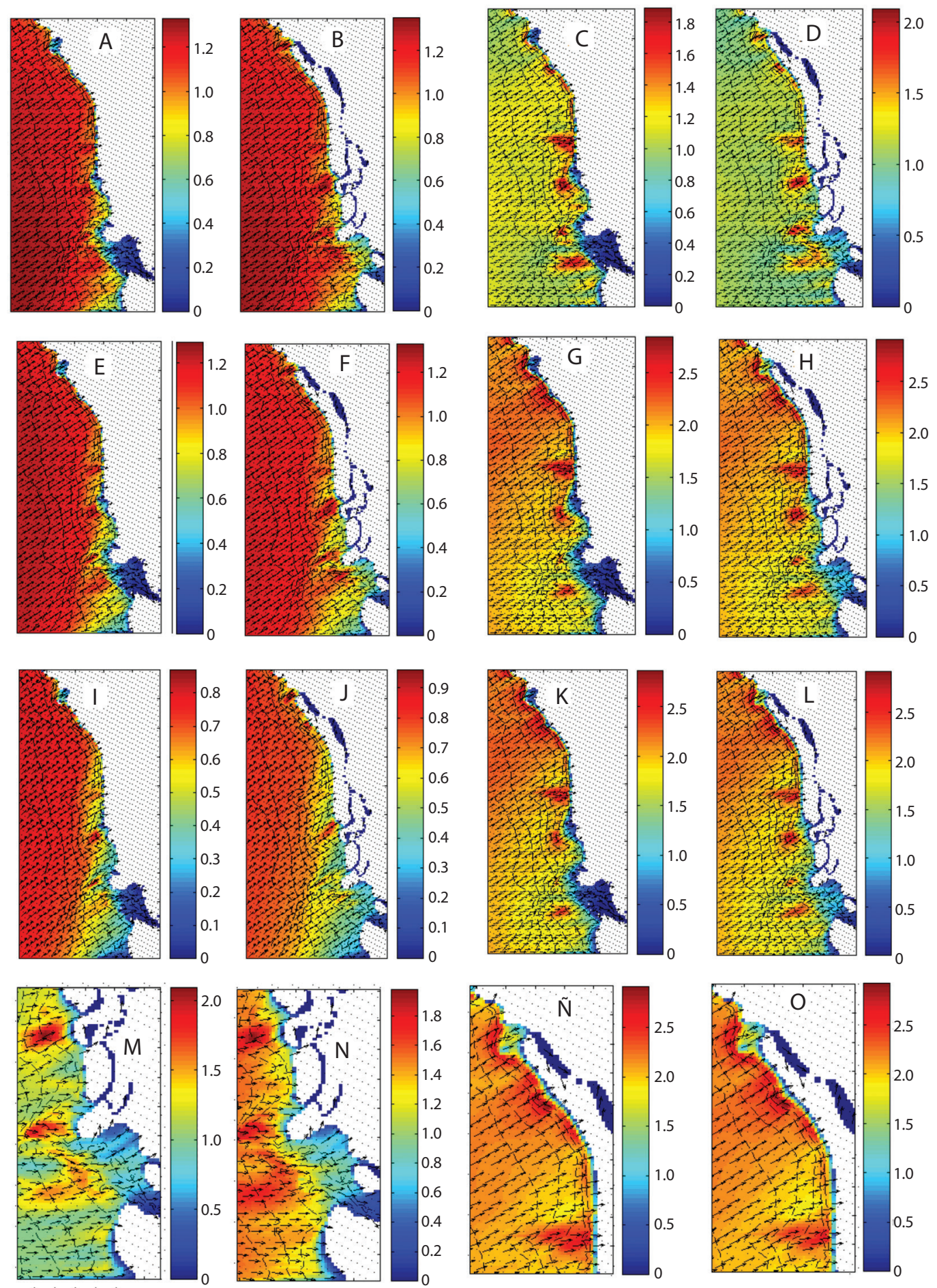
(Fig. 7B), rompe también sobre los bajos batimétricos, pero le queda energía (altura) para penetrar al interior de los mismos y alcanzar el borde costero, como se nota por ejemplo, en el interior de Bahía Zacate, donde aún puede haber olas con alturas de $0.6 \mathrm{~m}$. También tienen energía sobre la punta al sur de Boca Zacate, donde pueden darse olas hasta de $1 \mathrm{~m}$. Además, la condición de marejada de fondo de esa dirección (Fig. 7C y 7D), muestra una característica de oleaje de períodos largos $(\mathrm{T} p=18 \mathrm{~s})$ : las alturas de las olas aumentan conforme se aproximan a la orilla, y sus rompimientos se hacen más fuertes sobre los bajos batimétricos al frente de las bocas $(2.2 \mathrm{~m})$. Esta condición anterior es así tanto en marea baja, como en marea alta, excepto que en este último caso, aún hay energía de la ola hacia el interior de las bocas (hasta $0.8 \mathrm{~m}$ de altura de ola en Boca Zacate). Aunque el oleaje es de dirección oeste, se transforma por efectos de la batimetría costera, en oleaje con dirección del suroeste.

Dirección suroeste: Los casos más representativos de esta dirección se muestran en las Fig. 7E a $7 \mathrm{H}$.

Un resumen de los resultados desde esta dirección indican que los oleajes promedio
$(\mathrm{Hs}=1.6 \mathrm{~m})$ y periodos bajos $(\mathrm{Tp}=6.5 \mathrm{~s}$, Fig. $7 \mathrm{E}$ y 7F) pierden energía (altura) desde agua profunda y llegan como alturas menores de $1 \mathrm{~m}$ a la orilla. En la condición más extrema desde esta dirección (Fig. $7 \mathrm{H}$ ), las olas rompen con mayor energía (altura) sobre la región al norte, hacia Boca Coronado. Alturas sobre los $3 \mathrm{~m}$ se notan en Fig. $7 \mathrm{G}$ y $7 \mathrm{H}$, lo que indica que conforme aumenta el período de ola, mayor es el efecto de refracción (cambio de dirección) y mayor es la altura de la ola al romper sobre la costa. En marea alta, las olas rompen sobre los bajos (Fig. 7G), pero también parte de la energía de ellas continúa hacia el interior de Boca Zacate por ejemplo (Fig. 7H), y en la punta al sur de esta boca. La condición más extrema (Fig. $7 \mathrm{H}$ ) indica que pueden penetrar olas de hasta $1 \mathrm{~m}$ al interior de las bocas, y de hasta $1.5 \mathrm{~m}$ sobre la punta al sur de Boca Zacate.

Dirección sur: Las Fig. 7I a 7L muestran los resultados más representativos desde esta dirección.

Los resultados de este particular análisis muestra que el oleaje promedio del sur con períodos bajos $(\mathrm{T} p=6.2 \mathrm{~s})$ llega con la menor energía a esta región $(1 \mathrm{~m})$ sobre Boca Coronado $(<1 \mathrm{~m}$, Fig. 7I). Sobre la parte sur de la

Fig. 7. Distribución de la altura $(\mathrm{m})$ y dirección (flecha) en agua profunda en: 7A: marea baja y 7B: marea alta con oleaje del suroeste $\mathrm{Hs}=1.7 \mathrm{~m}, \mathrm{Tp}=6.4 \mathrm{~s}$, Viento $=5.7 \mathrm{~m} / \mathrm{s}$ y Direc. viento $=90^{\circ} ; 7 \mathrm{C}$ : marea baja y 7D: marea alta con oleaje del oeste $\mathrm{Hs}=1.7 \mathrm{~m}, \mathrm{Tp}=18.0 \mathrm{~s}$, Viento $=3.15 \mathrm{~m} / \mathrm{s}$ y Direc. viento $=90^{\circ} ; 7 \mathrm{E}$ : marea baja y $7 \mathrm{~F}:$ marea alta con oleaje del suroeste $\mathrm{Hs}=1.6 \mathrm{~m}$, $\mathrm{Tp}=6.5 \mathrm{~s}$, Viento $=4.5 \mathrm{~m} / \mathrm{s}$ y Direc. viento $=90^{\circ} ; 7 \mathrm{G}$ : marea baja y $7 \mathrm{H}$ : marea alta con oleaje del suroeste $\mathrm{Hs}=2.65 \mathrm{~m}, \mathrm{Tp}=20.0 \mathrm{~s}$, Viento $=11.0 \mathrm{~m} / \mathrm{s}$ y Direc. viento $=140^{\circ} ; 7 \mathrm{I}$ : marea baja y $7 \mathrm{~J}$ : marea alta con oleaje del sur $\mathrm{Hs}=1.2 \mathrm{~m}, \mathrm{Tp}=6.2 \mathrm{~s}$, Viento $=3.5 \mathrm{~m} / \mathrm{s}$ y Direc. viento=133 ; 7K: marea baja y $7 \mathrm{~L}$ : marea alta con oleaje del sur $\mathrm{Hs}=3.0 \mathrm{~m}, \mathrm{Tp}=20.0 \mathrm{~s}$, Viento=11.0m/s y Direc. viento $=134^{\circ} ; 7 \mathrm{M}$ : oleaje del suroeste $\mathrm{Hs}=2.65 \mathrm{~m}, \mathrm{Tp}=20.0 \mathrm{~s}$, Viento $=11.0 \mathrm{~m} / \mathrm{s}$ y Direc. viento $=140^{\circ}$; acercamiento en Boca Zacate. $7 \mathrm{~N}$ : oleaje del sur $\mathrm{Hs}=3.0 \mathrm{~m}, \mathrm{Tp}=20.0 \mathrm{~s}$, Viento $=11.0 \mathrm{~m} / \mathrm{s}$ y Direc. viento $=134^{\circ}$, acercamiento en Boca Zacate. $7 \tilde{\mathrm{N}}$ : oleaje del suroeste $\mathrm{Hs}=2.65 \mathrm{~m}, \mathrm{Tp}=20.0 \mathrm{~s}$, Viento $=11.0 \mathrm{~m} / \mathrm{s}$ y Direc. viento $=140^{\circ}$, acercamiento en Boca Coronado, y 70 : oleaje del sur $\mathrm{Hs}=3.0 \mathrm{~m}, \mathrm{Tp}=20.0 \mathrm{~s}$, Viento $=11.0 \mathrm{~m} / \mathrm{s}$ y Direc. viento $=134^{\circ}$, acercamiento en Boca Coronado (estás cuatro últimas simuladas con marea alta).

Fig. 7. Distribution of wave height $(\mathrm{m})$ and direction (arrow) in deep water in: 7A: high tide and 7B: low tide with waves from southwest $\mathrm{Hs}=1.7 \mathrm{~m}, \mathrm{Tp}=6.4 \mathrm{~s}$, Wind $=5.7 \mathrm{~m} / \mathrm{s}$ and Wind $\mathrm{dir} .=90^{\circ} ; 7 \mathrm{C}$ : low tide and 7D: high tide with waves from west $\mathrm{Hs}=1.7 \mathrm{~m}, \mathrm{Tp}=18.0 \mathrm{~s}$, Wind $=3.15 \mathrm{~m} / \mathrm{s}$ and Wind $\mathrm{dir} .=90^{\circ} ; 7 \mathrm{E}$ : low tide and $7 \mathrm{~F}$ : high tide with waves from southwest $\mathrm{Hs}=1.6 \mathrm{~m}$, $\mathrm{Tp}=6.5 \mathrm{~s}$, Wind $=4.5 \mathrm{~m} / \mathrm{s}$ y Wind $\operatorname{dir} .=90^{\circ} ; 7 \mathrm{G}$ : low tide and $7 \mathrm{H}$ : high tide with waves from southwest $\mathrm{Hs}=2.65 \mathrm{~m}, \mathrm{Tp}=20.0 \mathrm{~s}$, Wind $=11.0 \mathrm{~m} / \mathrm{s}$ and Wind dir. $=140^{\circ}$; 7I: low tide and $7 \mathrm{~J}$ : high tide with waves form south $\mathrm{Hs}=1.2 \mathrm{~m}, \mathrm{Tp}=6.2 \mathrm{~s}$, Wind $=3.5 \mathrm{~m} / \mathrm{s}$ and Wind dir. $=133^{\circ} ; 7 \mathrm{~K}$ : low tide and $7 \mathrm{~L}$ : high tide with waves from south $\mathrm{Hs}=3.0 \mathrm{~m}, \mathrm{Tp}=20.0 \mathrm{~s}$, Wind=11.0m $/ \mathrm{s}$ and Wind dir. $=134^{\circ} ; 7 \mathrm{M}$ : waves from southwest $\mathrm{Hs}=2.65 \mathrm{~m}, \mathrm{Tp}=20.0 \mathrm{~s}$, Wind $=11.0 \mathrm{~m} / \mathrm{s}$ and Wind dir. $=140^{\circ}$; focused on Boca Zacate. $7 \mathrm{~N}$ : waves from south $\mathrm{Hs}=3.0 \mathrm{~m}, \mathrm{Tp}=20.0 \mathrm{~s}$, Wind $=11.0 \mathrm{~m} / \mathrm{s}$ and Wind $\operatorname{dir} .=134^{\circ}$; focused on Boca Zacate. $7 \tilde{\mathrm{N}}$ : waves from southwest $\mathrm{Hs}=2.65 \mathrm{~m}, \mathrm{Tp}=20.0 \mathrm{~s}$, Wind $=11.0 \mathrm{~m} / \mathrm{s}$ and Wind $\operatorname{dir} .=140^{\circ}$, focused on Boca Coronado, and $7 \mathrm{O}$ : waves from south $\mathrm{Hs}=3.0 \mathrm{~m}, \mathrm{Tp}=20.0 \mathrm{~s}$, Wind $=11.0 \mathrm{~m} / \mathrm{s}$ and Wind $\mathrm{dir} .=134^{\circ}$, focused on Boca Coronado (the latest were simulated in high tide condition). 
región, Boca Zacate, la altura es de $0.6 \mathrm{~m}$ en marea baja sobre los bajos batimétricos, y $0.4 \mathrm{~m}$ en el interior de la bahía (Fig. 7I y 7J). La condición más extrema (Fig. 7L), muestra que el oleaje en este caso también, tiene mayor altura sobre la región norte de Boca Coronado $(\sim 3 \mathrm{~m})$. Se pueden producir olas con alturas de hasta de $1 \mathrm{~m}$ en el interior de Boca Zacate y $1.5 \mathrm{~m}$ en la punta al sur de Boca Zacate (Fig. 7L).

En general, este estudio indica que el oleaje más frecuente del oeste y suroeste (Fig. $7 \mathrm{M} \mathrm{y}$ $7 \mathrm{~N}$ ) ingresa hacia el interior de Bahía Zacate y sobre el manglar en sus alrededores con alturas que superan $1 \mathrm{~m}$. Adicionalmente se encuentra que oleaje del suroeste, y luego del sur (Fig. 7Ñ y 7O), muestran un patrón muy similar (mas no igual), con una mayor energía de oleaje (mayor altura) a lo largo de Playa Garza ( $>3 \mathrm{~m})$, lo que contribuye con la mayor dinámica de los sedimentos hacia Boca Coronado.

Aumento de la altura del oleaje: La Fig. 8 muestra los promedios y la tendencia de la serie mensual de las alturas de las olas frente al Pacífico Sur de Costa Rica. Los parámetros estadísticos Student $\mathrm{t}=5.6$ y razón de Fisher $\mathrm{F}=31$, indican que esta tendencia es positiva $\mathrm{y}$ significativa (Blank, 1980).
En las Figs. 9 y 10 se pueden observar los impactos en el manglar, principalmente sobre la región sur, donde se nota la muerte de los árboles y el retroceso en la cobertura del manglar.

Es claro en las Figs. 9 y 10, cómo el oleaje llega hasta el borde del manglar, modificando el tipo sedimento, siendo éste más arenoso que limo (lo cual está en relación con la energía de la ola a la cual están sometidos estos bordes). Pero también como demuestra la Fig. 10, el perfil del borde también se ha ido modificando, donde justamente por la energía de las olas, se está formando una playa arenosa con berma (lomo en la playa). El alcance de las olas hasta estas regiones, está asociado al aumento relativo del nivel del mar en la región, y a las modificaciones que continúan experimentando las bocas, siendo por ejemplo, Boca Zacate, más amplia con el tiempo, pues ha perdido parte de su tierra firme con los años sobre la punta al sur, y esto permite mayor ingreso de oleaje a su interior. Por esta misma razón, Isla Coco (Fig. 11), ha perdido más área en los últimos años.

Balance de áreas de las islas: El balance total de la cobertura o área de las islas, ha sido

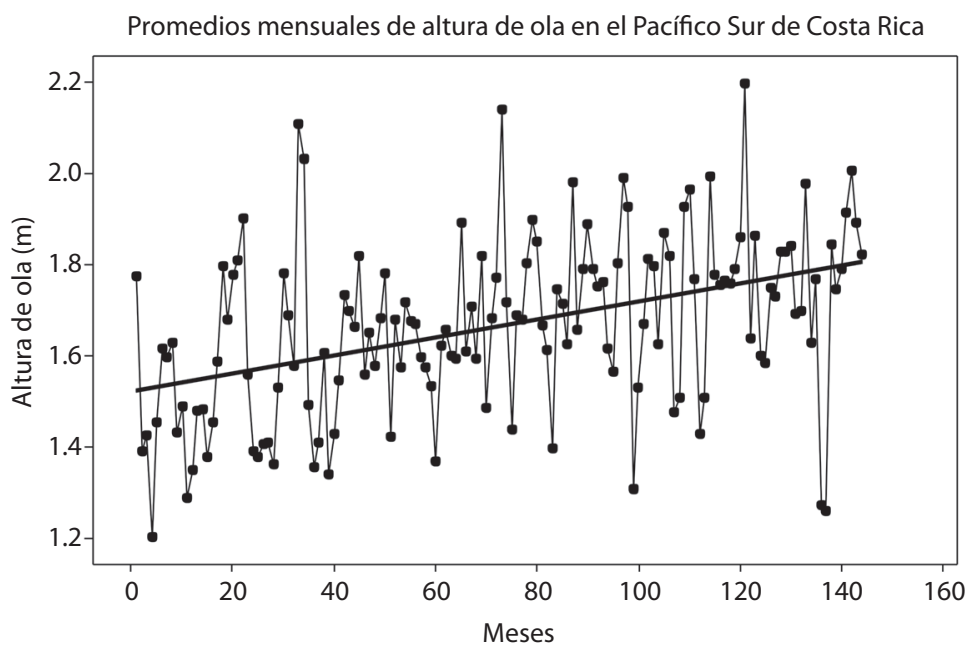

Fig. 8. Promedios mensuales de la altura de la ola (m) frente a la región del HNTS. Datos de la NOAA entre 1997-2008. Fig. 8. Monthly averages of wave height (m) in front of HNTS region. NOAA Data between 1997-2008. 


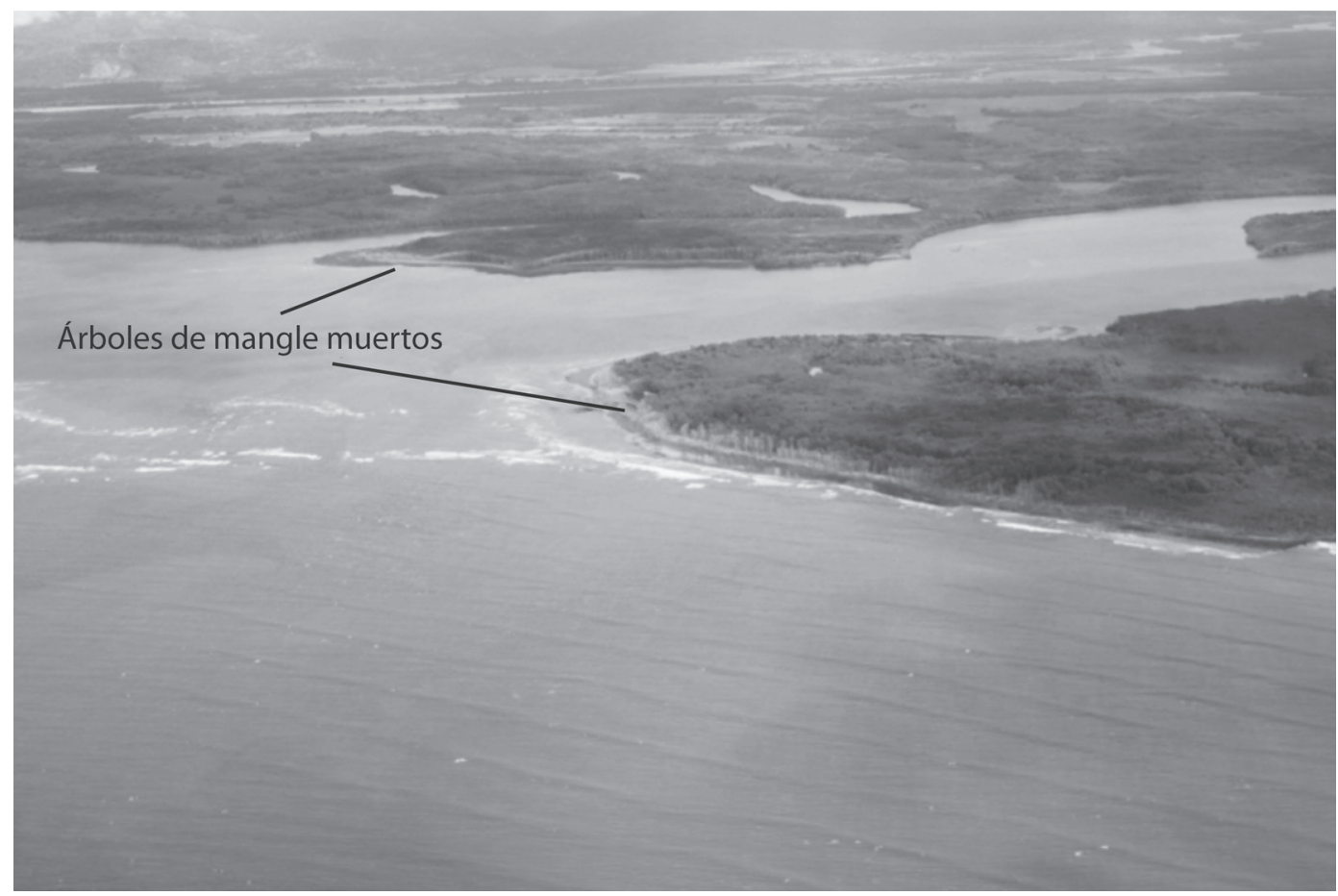

Fig. 9. Fotografía aérea de Isla Zacate e Isla Coco que muestran erosión.

Fig. 9. Aerial photograph of Isla Zacate and Isla Coco showing erosion.

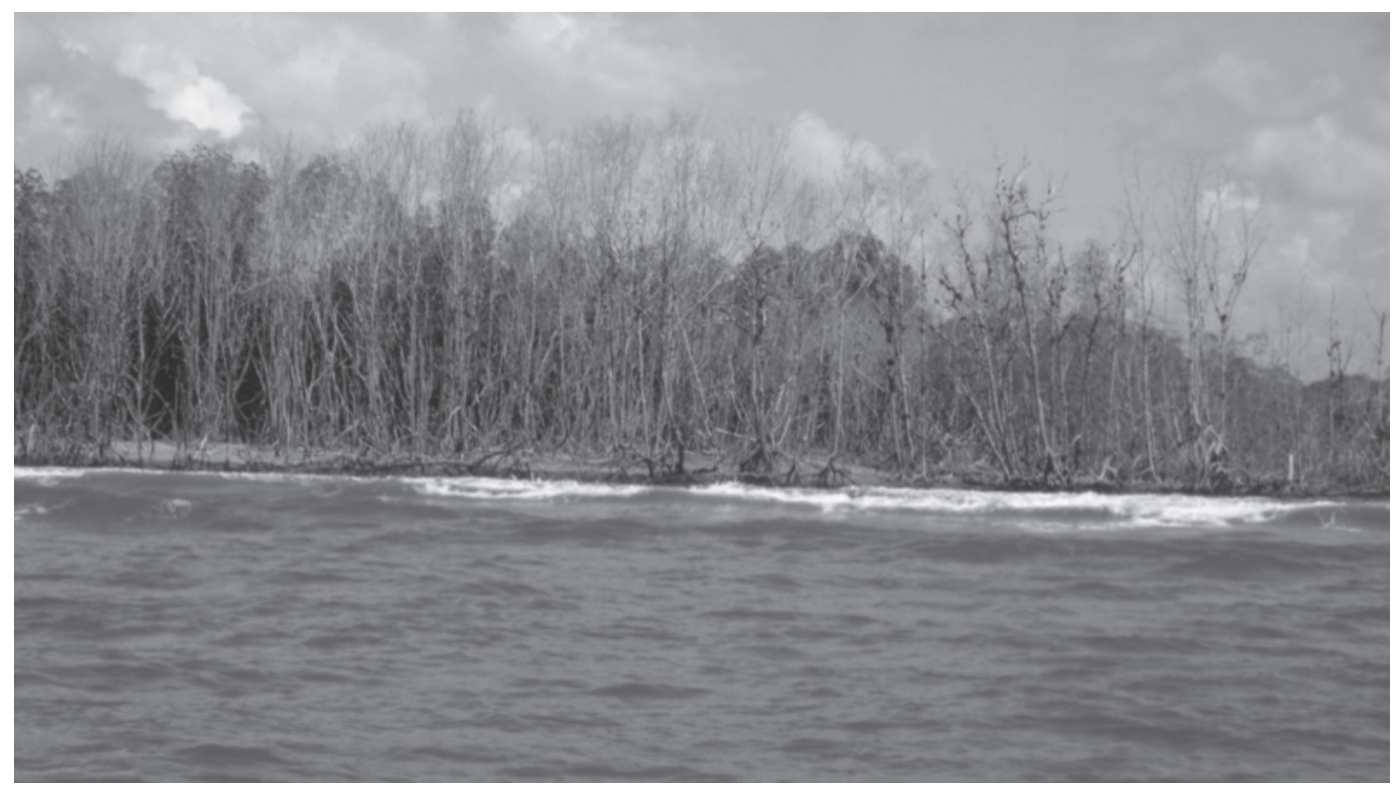

Fig. 10. Manglar en la punta al sur de Isla Zacate en marea alta el 31 de enero del 2011.

Fig. 10. Mangrove on the southern tip of Isla Zacate at high tide the January 31, 2011. 


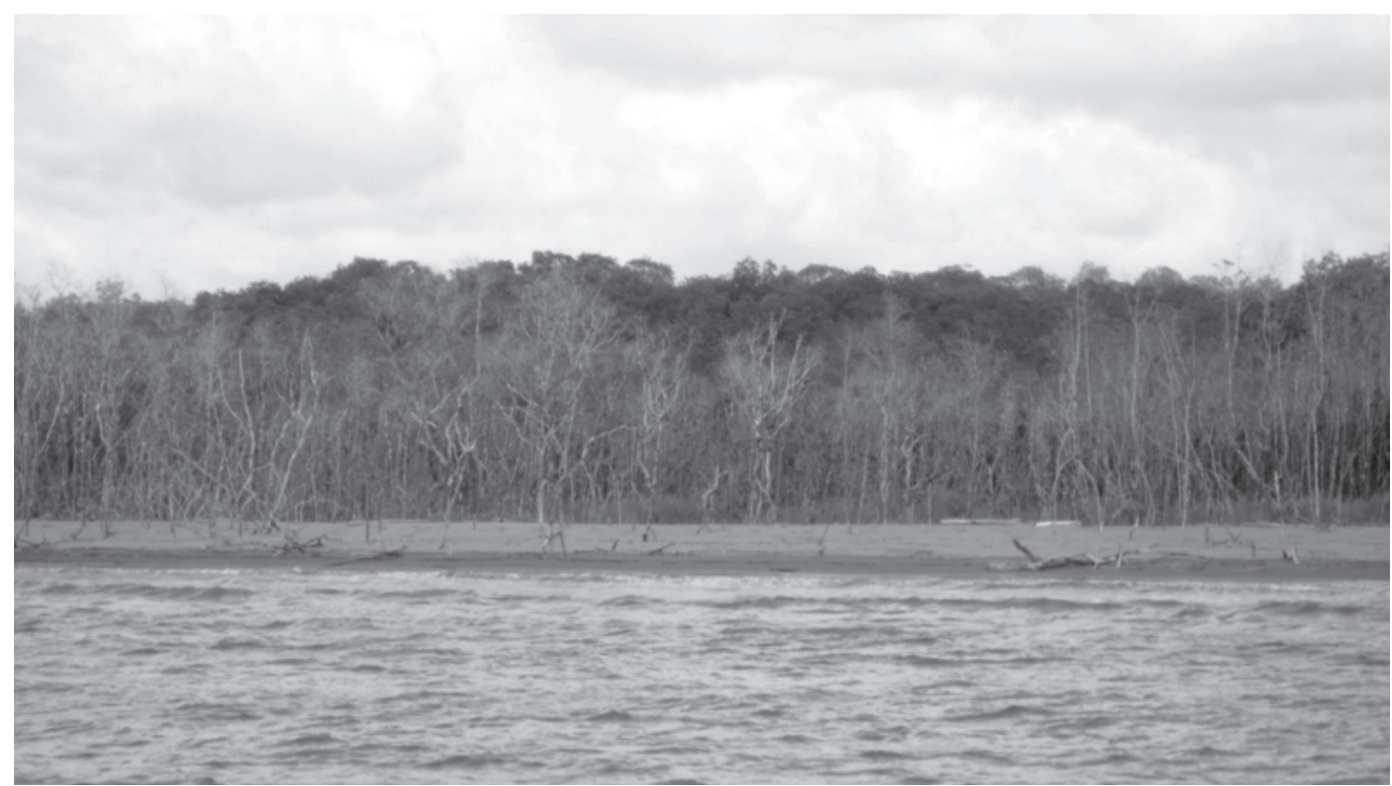

Fig. 11. Manglar en Isla Zacate en marea alta el 31 de enero del 2011.

Fig. 11. Mangrove in Isla Zacate at high tide the January $31^{\text {st }}, 2011$.

negativo desde 1948 (Cuadro 3: -1084900m²), al menos en las cinco islas que se estudiaron (Fig. 12). Es decir, el sistema de islas ha perdido más área, y por tanto sedimentos, de lo que ha ganado. Por ejemplo, la Isla 1 (Isla Playa Garza) entre los años 48 y 97 no aumentó tanto, como si lo hizo entre el 97 y 2005. Isla Zacate tuvo una pérdida mayor de área entre el 48 y 97, y aunque siguió perdiendo entre el 97 y el 2005, lo hizo más lentamente. El mismo patrón muestra la Isla Coco (Isla 5).

\section{DISCUSIÓN}

Definitivamente, la acción del oleaje genera la dinámica de los sedimentos en las bocas como Coronado, Nueva, Brava, Chica y Zacate, como lo demuestra este estudio. El aumento relativo del nivel del mar en los últimos años, ha permitido el desarrollo de una nueva plataforma para el oleaje, cuya energía penetra más adentro de las bahías y bocas. Esto sucede justamente durante las mareas más altas, el oleaje

\section{CUADRO 3}

Balance de áreas $\left(\mathrm{Km}^{2}\right)$ en las principales islas al frente de la región HNTS

TABLE 3

Budget of areas $(\mathrm{km} 2)$ on the main islands in front of the HNTS region

\begin{tabular}{cccc} 
Isla & Período 48-97 & Período 97-05 & Período 48-2005 \\
1 & +0.7796 & +2.4401 & +32197 \\
2 & +0.9053 & -0.2168 & +0.6885 \\
3 & -0.4495 & -0.0001 & -0.4496 \\
4 & -0.8652 & +0.1999 & -0.6653 \\
5 & -1.1851 & -0.0927 & -1.2778 \\
6 & -2.5921 & -0.0012 & -2.6004 \\
Balance total & -3.4070 & -2.3292 & -1.0849 \\
\hline
\end{tabular}

\footnotetext{
“+”: ganancia de área. “-”: pérdida de área.
} 


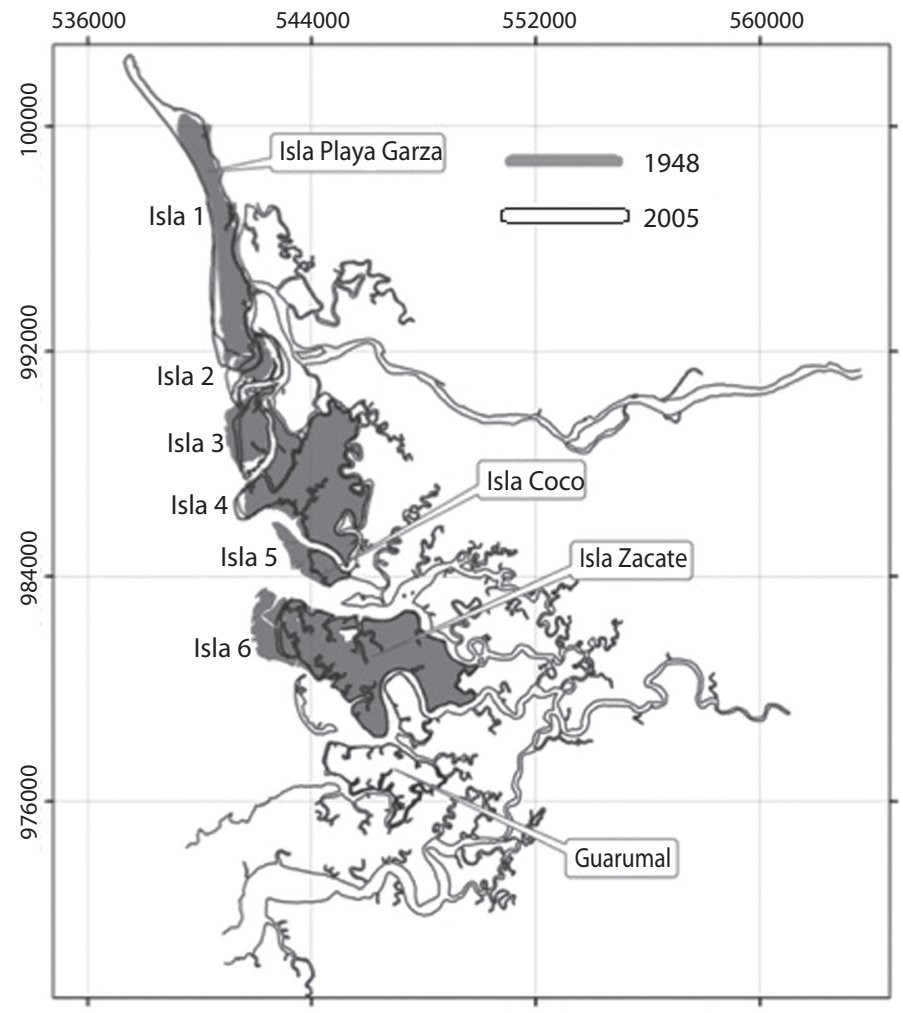

Fig. 12. Principales islas con cambios en la región del HNTS. Isla $1=$ =Isla Barrera Playa Garza, Isla 5=Isla Coco (Ortíz, 2008), Isla 6=Isla Zacate (Ortíz, 2008).

Fig. 12. Main islands with changes at the HNTS region. Island 1=Isla Barrera Playa Garza, Island 5=Isla Coco (Ortíz, 2008), Island 6=Isla Zacate (Ortíz, 2008).

alcanza el borde del manglar, lo que produce un lavado del sedimento fino (lodos), quedando sedimentos más gruesos (arenas), un sustrato que no favorece el crecimiento del manglar (Jiménez, 1994). A la vez, el oleaje modifica el perfil del borde del manglar formando una berma (Komar, 1976) con sedimento más grueso, lo cual es una característica geomorfológica en una playa que tiene energía de olas (Komar, 1976).

El fenómeno de mareas astronómicas altas se repite varias veces al año, y además, es más frecuente durante algunos años del ciclo de mareas extraordinarias (Lizano, 1997; 2001). Adicionalmente, con frecuencia que podría estar aumentando (Grant, 1981; Fetzek, 2009), se tienen fenómenos adicionales que aumentan el nivel del mar como el fenómeno El Niño. A esto se suma la posibilidad de tener tormentas de viento locales y efectos indirectos de huracanes del Caribe, que además de apilamiento de agua sobre la costa por el viento, generan olas de alturas significativas, y que cuando coinciden con mareas altas, las olas aceleran los procesos de erosión.

Adicionalmente se encuentra que el oleaje ha estado aumentando en altura en los últimos años en esta región, una consecuencia también, del calentamiento global. Aunque la serie analizada corresponde a un período corto, la tendencia positiva es significativa estadísticamente, y es coherente con la observación de otras variables océano-meteorológicas a nivel mundial (Young et al., 2011). 
Esta zona es una región tectónicamente activa (Hebbeln et al., 1996; Espinoza et al., 2005), y aunque no hay evidencia de algún fenómeno reciente, es posible que se tengan efectos de la subducción de placas en ese aumento relativo del mar. Estudios de Cárdenes (2003) en Parrita indica que el aumento relativo del nivel del mar puede deberse a un posible basculamiento o hundimiento de origen tectónico. Un hundimiento similar en la costa de Guanacaste ha sido documentado por Norabuena et al. (2004) y Protti (2007), justamente después del terremoto de Guanacaste en el 2011, donde se produjeron levantamientos mayores de $50 \mathrm{~cm}$ en esta zona (Marino Protti, com. pers.), y hace pensar que el proceso de erosión en esta región del HNTS no es local. Las observaciones realizadas en las zonas vecinas, muestran procesos de erosión en playas y lugares al sur de esta región, y en la misma Península de Osa, como en la salida del Río Sierpe, Bahía Drake y San Pedrillo. También hay erosión en las playas del noroeste de la desembocadura del Río Grande de Térraba, como en playas Piñuelas, Ballena y Hermosa Sur. Además, como se ha reiterado una y otra vez, este es un problema generalizado en toda la costa de Costa Rica, que como lo menciona Lizano $(2010,2013)$, es un fenómeno asociado al aumento relativo del nivel del mar, con bastante seguridad ligado al calentamiento global, y con alguna posibilidad, al basculamiento de la costa.

La dinámica de sedimentos en islas barrera del Pacífico de Costa Rica se han estado dado desde años atrás, como lo demuestra el estudio de Lizano (2013) en Isla Damas y Palo Seco, y en la punta de Puntarenas por estudios de Denyer et al. (2004). Dinámica similar experimenta esta región del HNTS, como lo demuestra la serie temporal de los contornos de las islas que se estudiaron en esta ocasión hasta el 2005. El balance total de sedimentos desde 1948 hasta el 2005 , ha sido negativo $\left(-1084900 \mathrm{~m}^{2}\right)$. Este sedimento ha sido redistribuido hacia el interior de las bahías (como en Boca Zacate), como también lo menciona Ortiz (2008), o aumentando o creando otras islas barreras, como en Playa Garza. Este proceso comenzó hace años, al menos desde 1960, como lo indica el estudio Acón (2007). No se tienen datos de los últimos siete años, en los cuales se ha notado más cambio en todas las costas del Pacífico de Costa Rica (Lizano, 2013), pero de los procesos de erosión tan severos que se notaron durante el 2011, es posible que esta región entrara en otro período de intensificación de erosión costera.

El gran aporte de sedimentos del Río Grande de Térraba, ha mantenido relativamente estable de erosión toda la región norte del HNTS, en la cual más bien, la Isla Barrera Playa Garza ha crecido con el tiempo. Sin embargo, sobre el sector sur, con el menor aporte de sedimentos del Río Sierpe, se está produciendo un retroceso del sistema estuarino (Kjerfve, Michener \& Gardner, 1994), y este retroceso es un indicativo de un aumento relativo en el nivel del mar. Cambios seguirán ocurriendo en este sistema deltaico, en respuesta a un proceso que ya no está en equilibrio, como consecuencia de fuentes de sedimento y de aumento relativo del nivel del mar.

\section{AGRADECIMIENTOS}

Este estudio se realizó a través de una consultoría para el Proyecto Hidroeléctrico El Diquís, en el Humedal Nacional TérrabaSierpe del Instituto Costarricense de Electricidad (ICE). A Jorge Picado del P.H. El Diquís y José Alberto Zúñiga del Departamento de Hidrología del ICE por el apoyo en el trabajo de campo.

\section{RESUMEN}

Se estudió la dinámica de las mareas y el oleaje frente al Humedal Nacional Térraba-Sierpe (HNTS) y su relación con los procesos de erosión y la muerte del manglar en esta región. Se encuentra que un aumento relativo del nivel del mar, ya sea generado por el basculamiento de las costas, o por calentamiento global, es el responsable de crear una nueva plataforma para el oleaje y está modificando el perfil de la costa y cambiando el tipo de sedimento sobre el cual crece el manglar. Una mayor deposición de sedimentos sobre la región norte de este delta, mantiene el equilibrio de este sistema en esta zona, donde más bien hay una ganancia de sedimentos en la isla al frente de la desembocadura del Río Grande de Térraba. Un menor aporte de sedimentos 
del Rio Sierpe está generando una migración tierra adentro de este sistema estuarino frente a esta región, lo cual es un síntoma de un aumento relativo del nivel del mar. Los escenarios futuros sobre una mayor energía del oleaje, y de un continuo aumento del nivel del mar, no permitirán un equilibrio en la dinámica de los sedimentos, especialmente sobre la parte sur de este delta, por lo que continuará perdiendo la cobertura del manglar en este sistema estuarino.

Palabras clave: Erosión costera, aumento del nivel del mar, manglar, Térraba-Sierpe, modelo de olas.

\section{REFERENCIAS}

Acón, M. J. (2007). Perdida del Bosque de Manglar del sector de Boca Zacate del Humedal Nacional Térraba Sierpe del año 1960 al 2007. (Informe Final del Taller de Investigación en Manejo y Protección de los recursos naturales). Costa Rica: Universidad Estatal a Distancia.

Alfaro, E. J. (2007). Escenarios climáticos para temporadas con alto y bajo número de huracanes en el Atlántico. Revista de Climatología, 7, 1-13.

Amador, J., Chacón, E., \& Lizano, O. G. (1994). Estudio de efectos geofísicos del Terremoto de Limón mediante percepción remota y análisis hidrometeorológico. Revista Geológica América Central, (Vol. Esp.), 153-170.

Anónimo. (2006). Estrategia para el Desarrollo Sostenible de la Región Brunca, CR-TI007 (Informe Final, Documento No. 3). Costa Rica.

Anónimo. (2010). Maximum height of extreme waves up dramatically in Pacific Northwest. Science Daily. Retrieved from www.sciencedaily.com/releases/2010/01/100125123233.htm

Anónimo. (2012). Alianza clima y desarrollo. La gestión de riesgos de eventos extremos y desastres en América Latina y el Caribe. Aprendizajes del Informe Especial (SREX) del IPCC. Retrieved from www.cdkn.org/ srex

Blank, L. (1980). Statistical procedures for engineering, management and science. New York: McGraw-Hill Bood Company.

Booij, N., Ris, R. C., \& Holthuijsen, L. H. (1999). A third generation wave model for coastal regions: 1 . Model description and validation. Journal of Geophysical Research, 104, 7649-7666.

Cárdenes, G. (2003). Evolución de los sistemas sedimentarios costero y aluvial de la región de Parrita, Pacifico Central de Cota Rica. Revista Geológica de América Central, 28, 69-76.

Cooper, M. J. P., Beevers, M. D., \& Oppenheimer, M. (2008). The potential impacts of sea level rise on the coastal region of New Jersey, USA. Climatic Change, 90, 475-492.

Cortés, J., Soto-Soto, R., \& Jiménez, C. (1994). Efectos ecológicos del terremoto de Limón. Revista Geológica de América Centra, (Vol. Esp.), 187-192.

Delgado, D., \& Arguedas, C. (7 de setiembre, 2012). Capa rocosa atenuó fuerza destructiva del terremoto. $\mathrm{La}$ Nación. Disponible en http://www.nacion.com/201209-07/ElPais/capa-rocosa-atenuo-fuerza-destructivadel-terremoto.aspx?Page $=3$

Denyer, P., Arias, O., \& Personius, S. (1994). Efecto tectónico del terremoto de Limón. Revista Geológica de América Central, (Vol. Esp.), 39-52.

Denyer, P., Cárdenes, G., \& Kruse, S. (2004). Registro histórico y evolución de la barra arenosa de Puntarenas, Golfo de Nicoya, Costa Rica. Revista Geológica de América Central, 31, 45-59.

Dickson, M. E., Walkden, M. J. A., \& Hall, J. W. (2007). Systemic impacts of climate change on an eroding coastal region over the twenty-first century. Climatic Change, 84, 141-166.

Espinoza, J., Rojas, E., Solís, L., Aguilar, J., Gutiérrez, V., Granados, G., \& Rodríguez, H. (2005). Análisis Sedimentológico del delta Térraba (Costa Rica). Costa Rica: Escuela Centroamericana de Geología, Universidad de Costa Rica.

Fetzek, S. (2009). Impactos Relacionados con el Clima en la Seguridad Nacional en México y Centroamérica. Great Britain: Royal United Services Institute, Stephen Austin \& Sons.

Gornitz, V. (1995). Monitoring sea level changes. Climate Change, 31, 514-544.

Grant, P. J. (1981). Recently increased tropical cyclone activity and inferences concerning coastal erosion and inland hydrological regimes in New Zealand and Eastean Australia. Climate Change, 3, 317-332.

Hebbeln, D., Beese, D., \& Cortes, J. (1996). Morphology and sediment structures in Golfo Dulce, Costa Rica. Revista de Biología Tropical, 44(Suppl. 3), 1-10.

Jiménez, J. A. (1994). Los manglares del Pacifico centroamericano. Heredia, Costa Rica: Fundación UNA.

Kjerfve, B., Michener, W. K., \& Gardner, L. R. (1994). Impacts of climate change in estuary and delta environments. Gland, Switzerland: International Union for the Conservation of Nature and Natural Resources.

Knutson, T. R., Tuleya, R. E., \& Kurihara, Y. (1998). Simulated Increase of Hurricane Intensities in a CO2Warmed Climate. Science, 279, 1018-1020.

Komar, P. D. (1976). Beach Processes and Sedimentation. New Jersey: Prentice-Hall. 
Leatherman, S. P. (1977). Island States at Risck: Golbal Climate Change, Develpment and Population. Journal of Coastal Research, 24, 242.

Lizano, O. G. (1997). Las mareas extraordinarias de 1997 en la costa Pacífica de Costa Rica. Tópicos Meteorológicos y Oceanográficos, 4(2), 169-179.

Lizano, O. G., \& Salas, D. M. (2001). Variaciones geomorfológicas de la Isla Damas, Quepos en los últimos 50 años. "Ecosistemas Acuáticos de Costa Rica". Revista de Biología Tropical, 49(Supl. 2), 171-177.

Lizano, O. G., Ocampo, F. J., Alvarado, L. F., Puig, J. M., \& Vega, R. (2001). Evaluación de modelos numéricos de Tercera Generación para el pronóstico de oleaje en Centroamérica y México. Tópicos Meteorológicos y Oceanográficos, 8(1), 40-49.

Lizano, O. G. (2006). Algunas características de las mareas en la costa Pacífica y Caribe de Centroamérica. Ciencia y Tecnología, 24, 51-64.

Lizano, O. G. (2007). Climatología del viento y oleaje frente a las costas de Costa Rica. Ciencia y Tecnología, 25(1-2), 43-56.

Lizano, M., \& Lizano, O. G. (2010). Creación de escenarios de inundación en la Ciudad de Puntarenas ante el aumento del nivel del mar. InterSedes, XI(21), 215-229.

Lizano, O. G., \& Gutiérrez, A. (2011). Erosión en las costas de Costa Rica, un problema de todos. En Torno a la Prevención, 7, 14-16.

Lizano, O. G. (2013). Erosión en las playas de Costa Rica, incluyendo la Isla del Coco. InterSedes, 27(XIV), 6-27.

Miller, P. (2012). Weather gone wild. National Geographic, $222,30-53$

Mokrech, M., Nicholls, R. J., Richards, J. A., Henriques, C., Holman, I. P., \& Shackley, S. (2008). Regional impact assessment of flooding under future climate and socio-economic scenarios for East Anglia and MorthWest England. Climate Change, 90, 31-55.
Nicholls, R. J., \& Cazenave, A. (2010). Sea-Level Rise and Its Impact on Coastal Zones Science, 328, 1517-1520.

Norabuena, E., Dixon, T. H., Schwartz, S., DeShon, H., Newman, A., Protti, M., ... Sampson, D. (2004). Geodetic and seismic constraints on some seismogenic zone processes in Costa Rica. Journal of Geophysical Research, 109, 1-25.

Ortíz, E. (2008). Cambios geoformológicos en el litoral Caribe y Pacífico de Costa Rica. Caso del Complejo Déltico de Sierpe. Kurú: Revista Forestal, 5(15), $1-10$.

Pielke, R. A., Landsea, C. W., Mayfield, M., Laver, J., \& Pasch, R. (2005). Hurricanes and Global Warming. Bulletin of the American Meteorological Society, 86, 1571-1575.

Protti, M., Güendel, F., \& Malavassi, E. (2001). Evaluación del potencial sísmico de la Península de Nicoya. Heredia, Costa Rica: Editorial Fundación UNA.

Protti, M. (24 de julio, 2007). Límite dinámico y cambiante. La Nación. Disponible en http://wvw.nacion.com/ ln_ee/2007/julio/24/opinion1106780.html

Reyes, V., Miranda, M., Monge, C., \& Salas, F. (2007). Valoración económica del ecosistema Humedal Nacional Térraba-Sierpe y propuesta de mecanismos para su sostenibilidad, Costa Rica. In R. Ulate \& J. Cisneros (Eds.), Valoración Económica Ecológica y Ambiental. Análisis de casos en Iberoamérica (pp. 509-520). Costa Rica: Universidad Nacional.

Sorensen, R. M. (1978). Basic Coastal Engineering. New York: John Wiley \& Sons.

Young, I. R., Zieger, S., \& Bahanin, A. V. (2011). Global trends in wind speed and wave height. Science, 332, 451-455.

Zamora, P. (2006). Manglares de Costa Rica. In V. N. Muñoz \& M. A. Quesada (Eds.), Ambientes marino costeros de Costa Rica (pp. 23-40). Costa Rica: Conservation Internacional. 